\title{
Hemoglobin A1c Increased
}

National Cancer Institute

\section{Source}

National Cancer Institute. Hemoglobin A1c Increased. NCI Thesaurus. Code C122507.

A laboratory test result demonstrating an increase in the glycosylated hemoglobin in a blood sample. 